Technological University Dublin

\title{
A modified Langevin-Debye model for investigating the electro- optic behaviour of de Vries smectic liquid crystals
}

Vigneshwaran Swaminathan

Trinity College Dublin, Ireland

Vitaly Petrovich Panov

Trinity College Dublin, Ireland

Sithara Pavithran Sreenilayam

Trinity College Dublin, Ireland

See next page for additional authors

Follow this and additional works at: https://arrow.tudublin.ie/engscheleart2

Part of the Electrical and Computer Engineering Commons

\section{Recommended Citation}

V. Swaminathan, V.P. Panov, S.P. Sreenilayam, Yu. P. Panarin \& J.K. Vij (2019) A modified Langevin-Debye model for investigating the electro-optic behaviour of de Vries smectic liquid crystals, Liquid Crystals, 46:8, 1246-1251, DOI: 10.1080/02678292.2018.1549280

This Article is brought to you for free and open access by the School of Electrical and Electronic Engineering at ARROW@TU Dublin. It has been accepted for inclusion in Articles by an authorized administrator of ARROW@TU Dublin. For more information, please contact arrow.admin@tudublin.ie, aisling.coyne@tudublin.ie, gerard.connolly@tudublin.ie.

Funder: Science Foundation Ireland; United States National Science Foundation 


\section{Authors}

Vigneshwaran Swaminathan, Vitaly Petrovich Panov, Sithara Pavithran Sreenilayam, Yuri Panarin, and J. K. Vij

This article is available at ARROW@TU Dublin: https://arrow.tudublin.ie/engscheleart2/278 
See discussions, stats, and author profiles for this publication at: https://www.researchgate.net/publication/329560163

\section{A modified Langevin-Debye model for investigating the electro-optic behaviour of de Vries smectic liquid crystals}

Article in Liquid Crystals · December 2018

DOI: 10.1080/02678292.2018.1549280

\section{CITATIONS}

4

5 authors, including:

Digneshwaran Swaminathan

Vellore Institute of Technology Chennai Campus

15 PUBLICATIONS 80 CITATIONS

SEE PROFILE

Sithara Pavithran Sreenilayam

Dublin City University

36 PUBLICATIONS 367 CITATIONS

SEE PROFILE

Some of the authors of this publication are also working on these related projects:

Bistability \& Anchoring Energy of FLCs View project

Ferroelectric Liquid Crystals View project
Vitaly Petrovich Panov

Sungkyunkwan University

62 PUBLICATIONS 1,812 CITATIONS

SEE PROFILE

(6) Yu P Panarin

Technological University Dublin - City Campus

101 PUBLICATIONS 1,649 CITATIONS

SEE PROFILE 


\section{Liquid Crystals}

\section{A modified Langevin-Debye model for investigating the electro-optic behaviour of de Vries smectic liquid crystals}

\section{Swaminathan, V.P. Panov, S.P. Sreenilayam, Yu. P. Panarin \& J.K. Vij}

To cite this article: V. Swaminathan, V.P. Panov, S.P. Sreenilayam, Yu. P. Panarin \& J.K. Vij (2018): A modified Langevin-Debye model for investigating the electro-optic behaviour of de Vries smectic liquid crystals, Liquid Crystals, DOI: 10.1080/02678292.2018.1549280

To link to this article: https://doi.org/10.1080/02678292.2018.1549280

\section{曲 Published online: 10 Dec 2018.}

Submit your article to this journal $\pi$

Џ Article views: 27

View Crossmark data ¿ 


\title{
A modified Langevin-Debye model for investigating the electro-optic behaviour of de Vries smectic liquid crystals
}

\author{
V. Swaminathan ${ }^{a}$, V.P. Panov ${ }^{a}$, S.P. Sreenilayama , Yu. P. Panarin ${ }^{a, b}$ and J.K. Vij ${ }^{a}$

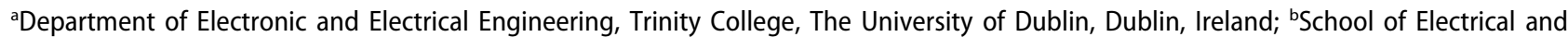 \\ Electronic Engineering, Dublin Institute of Technology, Dublin, Ireland
}

\begin{abstract}
An external electric field applied across a planar-aligned cell in Smectic $A^{*}$ phase of de Vries smectic liquid crystal induces director redistribution over a cone, resulting in a substantial increase in the birefringence and the apparent optical tilt angle. Such an electro-optic response is modelled by Shen et al. [Y. Shen et al., Phys. Rev. E 88, 062504 (2013)], who modified their previous hollow cone with a diffuse cone model by introducing the molecular distribution function limited over a range of tilt angles, that lie in between $\theta_{\min }$ and $\theta_{\max }$. The limits in these two tilt angles are assumed to be temperature independent though the tilt angle in between the two values can be temperature dependent. However, the high resolution measurements of birefringence and the layer thickness indicate the presence of temperature dependent diffuse cone angle in $\mathrm{SmA}^{*}$ phase.. In the proposed model, we replace $\theta_{\min }$ by $\theta_{T}$, a temperature dependent fitting parameter and the change shows that a better fit of the experimental data to the model is obtained. We determine the temperature dependence of $\theta_{\min }$ and show that this angle increases as $\mathrm{SmA}^{*}$ to $\mathrm{SmC}^{*}$ phase transition temperature is approached.
\end{abstract}
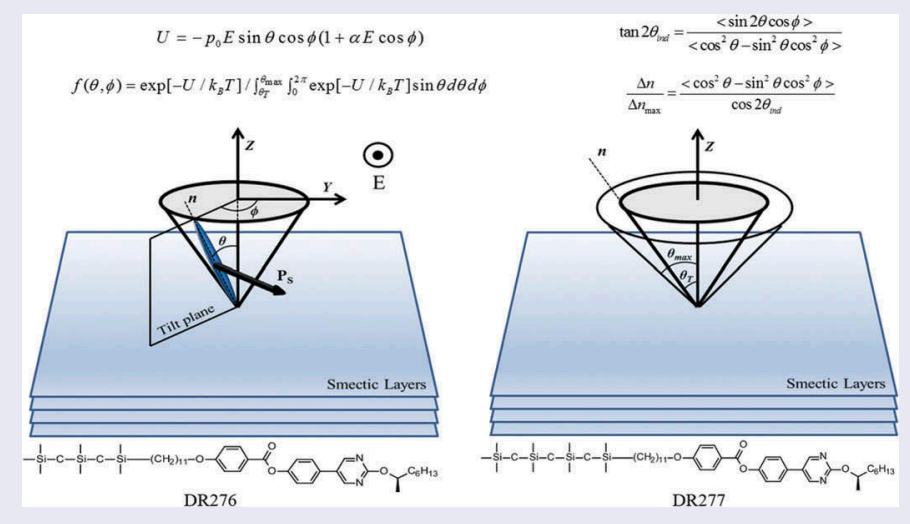

\section{ARTICLE HISTORY}

Received 7 September 2018

Accepted 13 November 2018

\section{KEYWORDS}

De Vries smectics; hollow cone model; diffuse cone model; ferroelectric liquid crystals; birefringence; apparent tilt angle

\section{Introduction}

The Smectic A $\left(\mathrm{SmA}^{\star}\right)$ to Smectic C $\left(\mathrm{SmC}^{\star}\right)\left(^{\star}\right.$ stands for the chiral molecules) phase transition in chiral smectic liquid crystals is associated with an emergence of a large tilt angle $\theta$ between the director and the layer normal, $\vec{k}$. The tilt angle in the $\mathrm{SmC}^{\star}$ phase can be as large as $\sim 45^{\circ}$ in orthoconic compounds, and the layer thickness in $\mathrm{SmC}^{\star}$ phase can thus be reduced up to $29 \%$ [1]. This is based on the assumption that the liquid crystalline molecules are approximated by inflexible hard rods, $d_{A C}=d_{A} \cos \theta ; d_{A C}$ and $d_{A}$ are the thicknesses of the smectic layer at the $\mathrm{SmA}^{*}-$ $\mathrm{SmC}^{\star}$ transition and in $\mathrm{SmA}^{*}$.

The advantage of using chiral molecules in a device is that spontaneous polarisation, $P$ s, emerges from a combination of the chirality and the dipole moment/s attached to (or close to) the chiral centre of the molecule. In $\mathrm{SmC}^{\star}$ phase, these dipoles produce $P$ s, which emerges at right angles to the tilt plane (the plane comprising the layer normal and the director), Ps interacts with the electric field and it produces a large torque on the director, which switches the device almost 1000 times faster [2] compared to a weaker torque exerted by the field on the dielectric anisotropy of nematics.

However, in a planar-aligned liquid crystal cell, the combined effects of layer shrinkage and the surface anchoring result in to the buckling of smectic layers in opposite directions thus to the formation of chevron structure/s [3]. The opposite folds of the chevron structures result in the appearance of the zigzag line defects. These defects severely 
degrade the optical quality of the display [4] and the contrast ratio is thereby reduced.

The zig-zag line defects in the texture are minimised if the layer shrinkage at the transition and within the SmC* phase is significantly reduced. In the ideal case, if the layer shrinkage is zero, then there should be no defects in the texture. Adrian de Vries [5-8] and Diele et al.[9] showed that some smectic liquid crystalline materials exhibit a little layer shrinkage at the SmA to SmC transition. Though these findings were made for smectics composed of achiral molecules, however results are equally applicable to chiral ones. One of the explanations for the reduced layer shrinkage may lie in that the layer thickness in SmA phase is much lower than length of the most stretched and plausible molecular configuration. As a consequence of it, the constituent molecules are tilted with a relatively large tilt angle in SmA phase but these are azimuthally distributed over a cone, as a consequence of $\mathrm{SmA}$ phase being uniaxial. At the SmA to SmC transition the azimuths condense to a single value of either 0 or $2 \pi$ rad due to a lift in the degeneracy of azimuthal angle, provided the tilt angle were not to change significantly. Hence the layer contraction at the transition temperature is almost close to zero.

The electro-optic response consists of measuring the (a) apparent tilt angle induced by the electric field and (b) the birefringence of a planar-aligned cell of cell-thickness of a few micrometres. The de Vries chiral smectics exhibit a characteristic sigmoidal shaped electro-optic response over a range of temperatures in the $\mathrm{SmA}^{*}$ phase [10-15]. In 2002, Clark et al. modelled the electro-optic response by the Langevin-Debye model with free energy function of the system such that the molecules in $\mathrm{SmA}^{*}$ phase are confined to a cone with a fixed tilt angle [10]. They demonstrated the fitting of the birefringence data with two values of the apparent tilt angle in $\mathrm{SmA}^{*}$ phase $\left(25^{\circ}\right.$ and $\left.34^{\circ}\right)$ as well their data in $\mathrm{SmC}^{*}\left(34^{\circ}\right)$ close to the $\mathrm{SmA}^{*}-\mathrm{SmC}^{*}$ transition temperature. This method can qualitatively reproduce many features of de Vries electro-optical behaviour, but the experimentally observed unique sigmoidal shape of the electro-optic response cannot be that easily fitted. To take account of it, Shen et al. in 2013 [11] suggested an alteration to this model to include the tilt over a limited range of tilt angles. This is termed as 'generalised Langevin-Debye model'. A detailed explanation of the above two models is given later in this article. In this work we modify the 'generalised Langevin-Debye model' as will be given below and modified model is used to fit the electro-optical response of the two chiral de Vries smectic liquid crystals investigated here.

\section{Material under investigation and the experimental techniques}

The molecular structure and the phase transition temperatures of the compounds DR276 and DR277 are given in Figure 1. The design of these molecules was inspired from structures of well-known de Vries smectic materials $\mathrm{MSi}_{3} \mathrm{MR} 11$ [16,17] and TSiKN65 [18]. The maximum layer shrinkage in $\mathrm{SmC}^{\star}$ measured using X-ray diffraction of DR276 and DR277 is found to be $\sim 1.9 \%$ and $0.9 \%$, respectively [19]. These materials have excellent LC characteristics in terms of exhibiting a wide range of temperatures of $\mathrm{SmA}^{\star}$ and $\mathrm{SmC}^{\star}$ phases. The temperature range for both also includes the room temperature, a highly desired practical characteristic of these materials for use in devices. Differential scanning calorimetry scans show $\mathrm{SmA}^{*}$ to $\mathrm{SmC}^{*}$ transition as weakly first order [19]. Here we use the experimental results of the field dependent birefringence and the apparent optical tilt angle in order to understand the microscopic origin of the features of the diffuse cone behaviour.

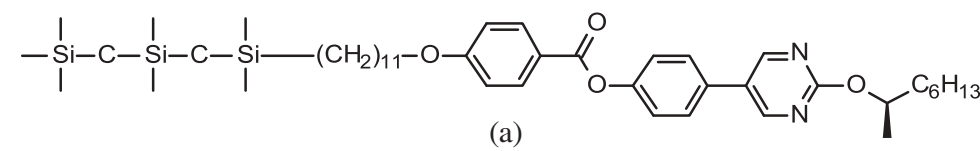

Cr $14^{\circ} \mathrm{C} \mathrm{SmC} * 78.5^{\circ} \mathrm{C} \mathrm{SmA} * 87^{\circ} \mathrm{C}$ Iso

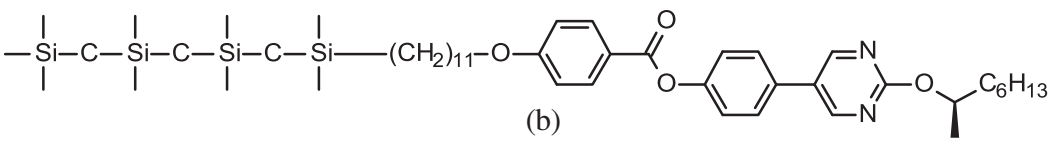

$\mathrm{Cr} 6{ }^{\circ} \mathrm{C} \mathrm{SmC} * 65.5{ }^{\circ} \mathrm{C} \mathrm{SmA} * 77{ }^{\circ} \mathrm{C}$ Iso

Figure 1. The molecular structure and the phase transition temperatures of (a) DR276 and (b) DR277. 
The birefringence and the optical tilt angle were measured as a function of the field using a rotating polarimetry technique explained in refs $[13,14,19,20]$.

\section{The electro-optic modelling}

Figure 2 shows the temperature dependent birefringence for zero and the maximum amplitude of the applied electric field applied across a planar-aligned cell with DR276 in $\mathrm{SmA}^{\star}$ phase [19]. For higher temperatures in the $\mathrm{SmA}^{*}$ phase (close to the isotropic phase), $\Delta n$ increases slightly by increase in the applied field. The birefringence for zero applied electric field decreases with temperature as opposed to normally increasing in a conventional smectic $A^{\star}$ phase. Furthermore on cooling in $\mathrm{SmA}^{*}$, the difference in the birefringence between the maximum and the zero fields increases significantly with a reduction in temperature reaching a maximum at the $\mathrm{SmA}^{*}-\mathrm{SmC}^{\star}$ transition. These are the typical characteristics of the de Vries $\mathrm{SmA}^{\star}$ [21]. These features are also observed in achiral de Vries smectics doped with a chiral dopant [22]. An applied electric field of $14 \mathrm{~V} / \mu \mathrm{m}$ increases the birefringence by $32 \%$ at the $\mathrm{SmA}^{*}-\mathrm{SmC}^{*}$ phase transition temperature $T_{A C}$. This feature reflects the emergence of molecular tilt angle within the temperature range of $S m A^{*}$, the angle first increases slowly in $\mathrm{SmA}^{\star}$ and then rapidly on approaching the $S m A^{\star}-$ $\mathrm{SmC}^{\star}$ phase transition temperature.

As outlined earlier, Clark et al. in 2002 showed that Langevin-Debye model could be used to qualitatively explain the electro-optic response of de Vries smectics by assuming that molecules are azimuthally distributed

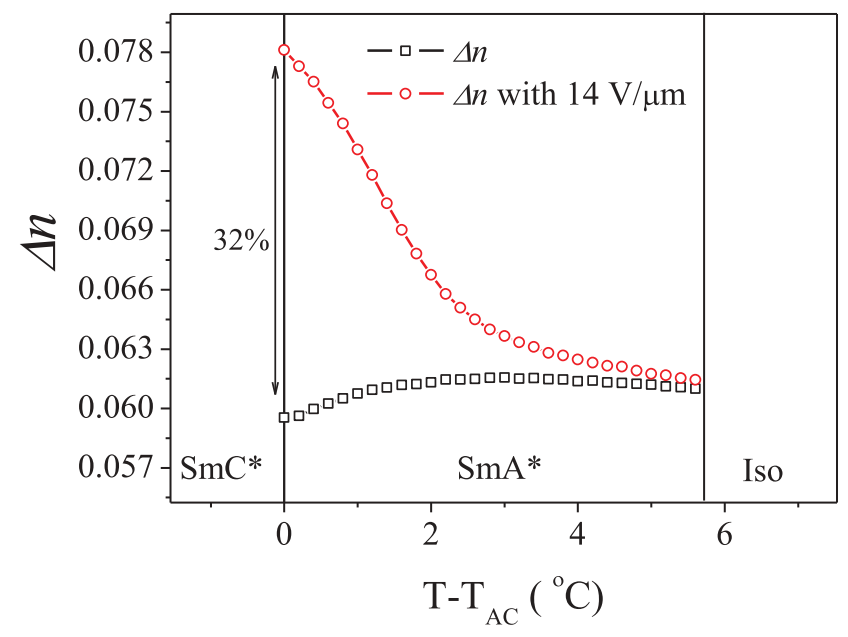

Figure 2. A plot of the birefringence $\Delta n$ of a planar-aligned LC cell measured as a function of the reduced temperature in the $\mathrm{SmA}^{*}$ phase with (i) electric field of $14 \mathrm{~V} / \mu \mathrm{m}$ applied across the cell and (ii) in the absence of electric field in $\mathrm{SmA}^{*}$ phase of DR276. over a cone with a fixed tilt angle [10]. When an electric field $E$ is applied along the plane of smectic layer, its interaction with the local dipole moment $(p)$ contributes to the free energy, $U=-p E \cos \phi$, $\phi$ is the angle between the dipole moment $p$ and the electric field $E$ across the cell. In order to quantitatively reproduce the sigmoidal response, Shen et al. in 2013 added a second term in $E$ to the free energy. In this case, the first is the linear term in $E,-p_{0} E \sin \theta \cos \phi$, the second order term in $E$ is $\alpha p_{0} E^{2} \sin \theta \cos ^{2} \phi$. These terms have degrees of freedom in both $\phi$ and $\theta . p=p_{0} \sin \theta$ is the dipole moment of the tilt correlated domain. $\theta(E)$ is restricted to vary within a range of values determined by the experiment. The free energy in the generalised Langevin-Debye model can thus be written as:

$$
U=-p_{0} E \sin \theta \cos \phi(1+\alpha E \cos \phi)
$$

$\alpha$ is the scaling factor for the second term, responsible for the sigmoidal response. In Shen's model $\theta(E)$ lies as already stated between $\theta_{\min }$ and $\theta_{\max }$. Both limits in the tilt angle are assumed to be temperature independent though the tilt angle in between the two values can be temperature dependent. In our model, we replace $\theta_{\min }$ by a temperature dependent variable $\theta_{T}$ and retain $\theta$ max as before. The molecular orientational distribution function (ODF) is defined with the lower limit in tilt $\theta_{T}$ as below:

$f(\theta, \phi)=\exp \left[-U / k_{B} T\right] / \int_{\theta_{T}}^{\theta_{\max }} \int_{0}^{2 \pi} \exp \left[-U / k_{B} T\right] \sin \theta d \theta d \phi$

An average of any parameter $\langle x\rangle$ is calculated from the given molecular distribution, such as:

$$
\langle x\rangle=\int_{\theta_{T}}^{\theta_{\max }} \int_{0}^{2 \pi} x(\theta, \phi) f(\theta, \phi) \sin \theta d \theta d \phi
$$

By averaging the dielectric tensor over the ODF and by neglecting the molecular biaxiality, Shen et al. derived the following formulae for the optical tilt $\theta_{\text {ind }}(E)$ and $\Delta n(E)$ as (for details see appendix Ref [11].),

$$
\begin{gathered}
\tan 2 \theta_{\text {ind }(E)}=\frac{\langle\sin 2 \theta \cos \phi\rangle}{\left\langle\cos ^{2} \theta-\sin ^{2} \theta \cos ^{2} \phi\right\rangle} \\
\frac{\Delta n(E)}{\Delta n_{\max }}=\frac{\left\langle\cos ^{2} \theta-\sin ^{2} \theta \cos ^{2} \phi\right\rangle}{\cos 2 \theta_{\text {ind }}}
\end{gathered}
$$

$\Delta n_{o}$ is the birefringence for zero field i.e. $(\mathrm{E}=0)$, for which $\theta_{\text {ind }}=0$. The optimal value of $\theta_{\min }$ is calculated from the ratio of the zero-field to the maximum field birefringence 
(saturated by the field) using Equation 8. $\theta_{\min }$ is replaced by $\theta_{T}$ and hence the limits of $\theta$ are from $\theta_{T}$ to $\theta_{\max }$ in Equation (6); $\theta_{\max }$ refers to the measured optical tilt angle for a large enough value of the applied electric field where the birefringence is saturated with field. For DR276, the measured saturating optical tilt angle $\theta_{\max }$ is $31^{\circ}$ and for DR277, the measured value of $\theta_{\max }$ is $33^{\circ}$ [19]. From the results of the temperature dependent layer thickness [Fig. 11 [19]] and the temperature dependence of the zero field birefringence $\Delta n_{o}$ (Figure 2) we anticipate that the lower limiting variable $\theta_{T}$ should be a function of temperature. For this reason, we modify the model and replace the lower integration limit $\theta_{\min }$ by $\theta_{T}$, as an additional fitting parameter in Equation (6), while $p_{0}$ and $\alpha$ are also the fitting parameters as before; $\theta_{T}, \alpha, p_{0}$ are found from Equations 7 and 8 which use Equations 4-6 through a computer programme written in MAPEL that best fits the plots of birefringence and the induced tilt angle both as functions of the electric field at a given temperature. The procedure so established is repeated for all experimental values of the temperature.

Figures $3(\mathrm{a}, \mathrm{b})$ shows the quality of the fit for the electric field dependent values for $\Delta n$ and $\theta_{i n d}$ for $T=\left(T_{A C}+1\right)^{\circ} \mathrm{C}$ in the $\mathrm{SmA}^{*}$ phase, respectively. The data for the induced tilt and birefringence (symbols) fits well to the model (solid lines) using Equations (7) and (8), respectively. We note that both $\Delta n$ and $\theta_{\text {ind }}$ show linear response for higher temperatures in the $\mathrm{SmA}^{\star}$ phase i.e. close to Iso$\mathrm{SmA}^{\star}$ transition temperature. Under slow cooling, the response particularly in birefringence exhibits a sigmoidal-shaped curve, clearly seen for $\left(T-T_{A C}\right) \leq 2^{\circ} \mathrm{C}[19]$. The modified generalised Langevin-Debye model reproduces the characteristic electro-optic response quite well supported by the quality of the fit. For the DR277, the theoretical curves are extrapolated up to $14 \mathrm{~V} / \mu \mathrm{m}$ to compare it with DR276 results.

The temperature dependence of the fitting parameters $p_{0}, \alpha$ and $\theta_{T}$ are shown in Figure 4. Magnitude of $p_{0}$ diverges on approaching the $\mathrm{Sm} A^{\star}-\mathrm{Sm} C^{\star}$ phase transition temperature, $p_{0}$ corresponds to the growth in the size of the tilt-correlated domain. Values of $p_{0}$ are very similar for both compounds DR276 and DR277, which implies that the size of the tilt-correlated domain in both cases is almost the same. However, if we compare $p_{0}$ with that obtained from the fitting of the other de Vries smectic materials, they differ and depend on different molecular systems. The phenomenological scaling parameter $\alpha$ increases almost linearly on cooling as $\mathrm{Sm} A^{*}-\mathrm{Sm} C^{\star}$ transition temperature is approached. The scaling parameter ' $\alpha$ ' for DR277 is greater than for DR276 by $10-20 \%$ as temperature is reduced. This is a consequence of DR277 exhibiting higher sigmoidality than DR276. For a given range of temperatures, $\theta_{T}$ is found to vary from $14^{\circ}$ to $18^{\circ}$ for DR276 and $24^{\circ}$

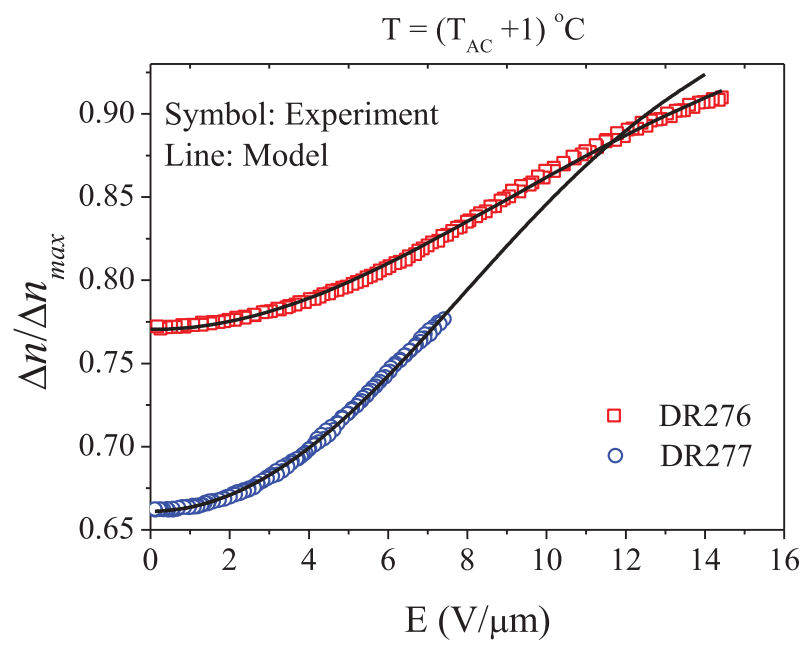

(a)

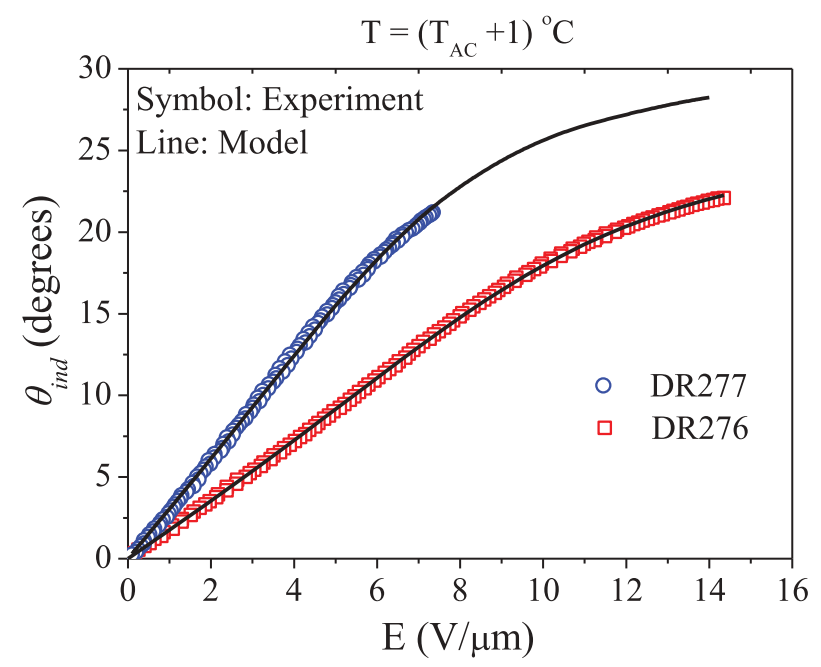

(b)

Figure 3. (a) Birefringence $\Delta n$ and (b) the apparent tilt angle $\theta_{\text {ind }}$ plotted as function of the applied electric field for $T=\left(T_{A C}+1\right)^{\circ} \mathrm{C}$ in the $\mathrm{SmA}^{*}$ phase. Symbols denote experimental data. The solid lines correspond to the fit of the data to the Modified model.

to $26^{\circ}$ for DR277, a clear increase is seen on cooling. This is found as would have been expected from the above discussion. Higher $\theta_{T}$ for DR277 supports the low layer shrinkage obtained from X-ray measurements and this also explains that birefringence for DR277 is lower than for DR276 at zero electric field.

Figure 5 shows the plots of ODF $f(\theta, \phi)$ for DR276 calculated from the modified generalised Langevin-Debye model for a temperature of $\left(T_{A C}+1\right)^{\circ} \mathrm{C}$ where amplitude of the applied field is varied. For zero field, the molecules are distributed evenly over a diffuse cone with $\theta$ varying from $\theta_{T}$ to $\theta_{\max }$. On applying the electric field across a planaraligned cell, the cone gets distorted as the molecules are forced to be restricted towards one of the sides of the cone. As the applied field is increased, molecular distribution $f(\theta$, 


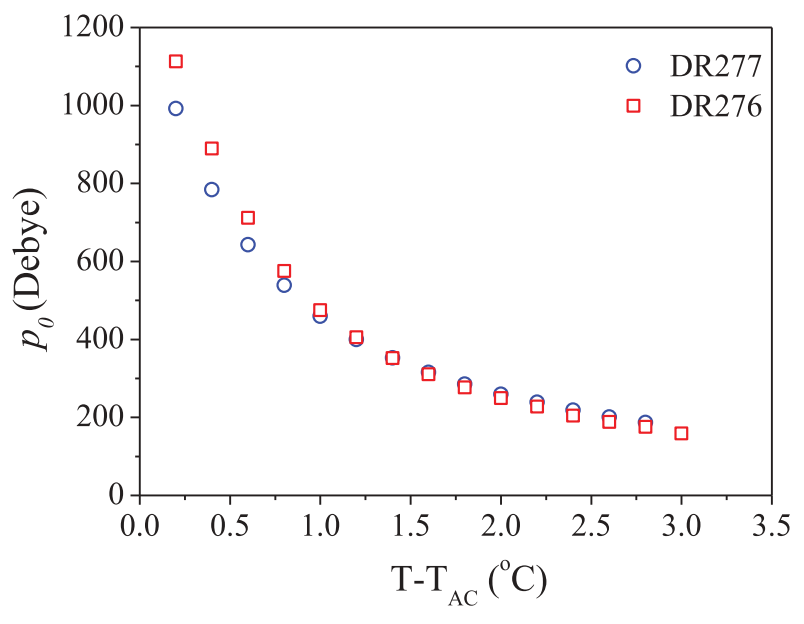

(a)

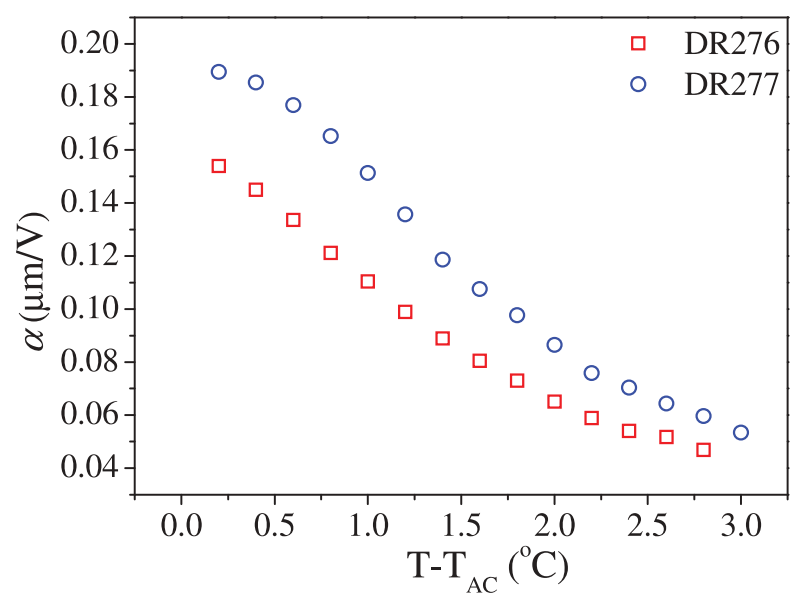

(b)

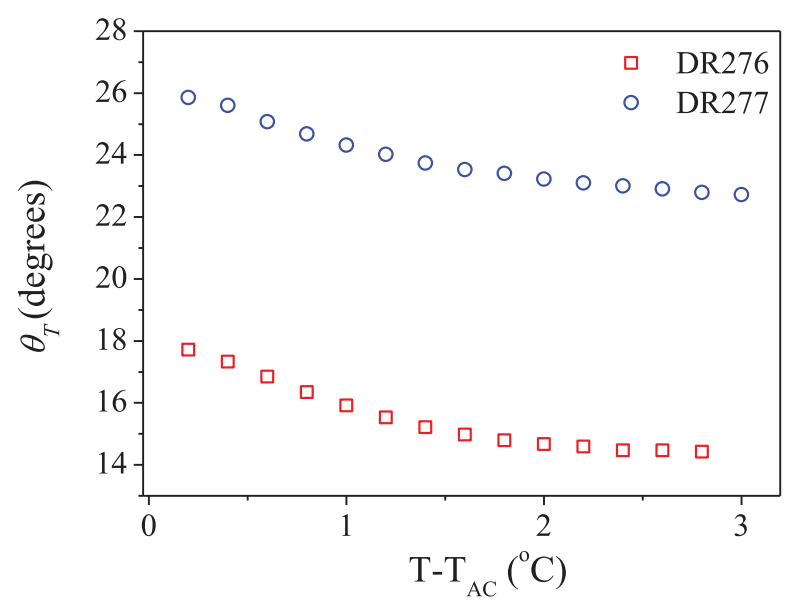

(c)

Figure 4. The temperature dependence of the fitting parameters for the compounds DR276 and DR277. (a) The local dipole moment $p_{0}$. (b) The phenomenological scaling parameter $a$, (c) The diffuse cone temperature dependent tilt angle $\theta_{T}$ of the model.

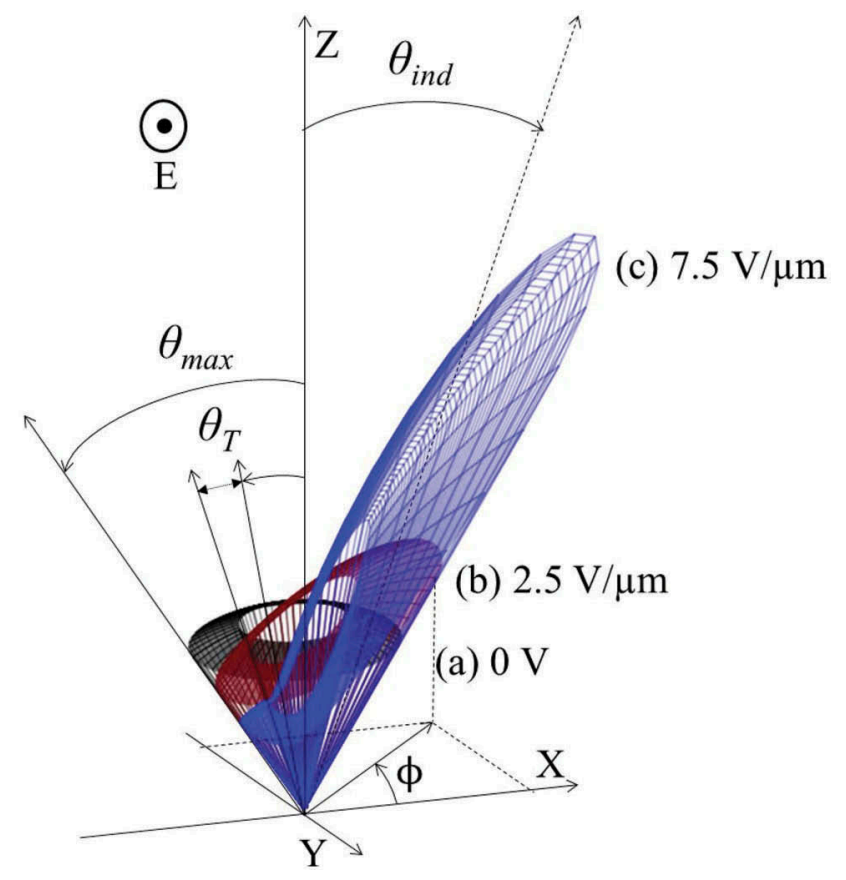

Figure 5. The orientational distribution function $f(\theta, \phi)$ calculated from the modified generalised Langevin-Debye model for DR276 for $\left(T_{A C}+1\right)^{\circ} \mathrm{C}$ at selected applied fields of (a) $0 \mathrm{~V} / \mu \mathrm{m}$, (b) $2.5 \mathrm{~V} / \mu \mathrm{m}$ and (c) $7.5 \mathrm{~V} / \mu \mathrm{m}$. (The distribution function is shown in the polar co-ordinates). The layer normal $\vec{k}$ is parallel to $Z$.

$\phi)$ is increasingly condensed where the molecules are confined to lie within a narrow range of azimuthal angles. This is also borne out by $\theta_{\text {ind }}$ saturating as observed experimentally.

\section{Conclusions}

The temperature dependence of the layer thickness and the birefringence exhibit unusual trends in de Vries $\mathrm{Sm} A^{*}$ phase compared to those of the conventional smectics. On this basis, it can be anticipated that the molecular tilt angle (apparent tilt angle) in $\mathrm{Sm} A^{\star}$ phase varies as a function of temperature. On following from it, we release the fixed variable $\left(\theta_{\min }\right)$ and make it temperature dependent $\left(\theta_{T}\right)$ in the generalised Langevin-Debye model of Shen et al. Excellent fits of the modified model to the experimental results for the two chiral de Vries liquid crystals are shown; the fit provides the basis that $\theta_{\min }$ is a temperature dependent variable. $\theta_{T}$ increases with a reduction in temperature and attains a maximum value at the $\mathrm{Sm} A^{\star}-\mathrm{SmC}^{\star}$ transition temperature, as expected. We show that the 'diffuse cone model' can satisfactorily explain the electro-optical behaviour of de Vries smectic liquid crystals. We reiterate our findings [19] that the compound with four carbosilanes is possibly one of the best ferroelectric liquid crystalline materials that satisfies the de Vries characteristics as well as 
its $\mathrm{SmC}^{\star}$ phase incorporates the room temperature down to $6^{\circ} \mathrm{C}$. We confirm that the birefringence data evidences the diffuse cone model, whereas the high resolution $\mathrm{x}$-rays data [23] can lead to the sugar loaf ODF. The solution to the apparent contradiction may lie in that the different techniques sense different parts of the molecule. The birefringence is mainly due to the mesogen (i.e. the core part) and $\mathrm{x}$-ray diffraction is due to the molecule or the hydrocarbon system as a whole [24].

\section{Disclosure statement}

No potential conflict of interest was reported by the authors.

\section{Funding}

This work was supported by the Science Foundation Ireland [13/ US/I2866] as part of the US-Ireland Research and Development Partnership programme jointly administered with the United States National Science Foundation under [grant number NSFDMR-1410649]. We thank W. J. Dowling for co-supervising 'VS' for his PhD degree of the University of Dublin.

\section{References}

[1] Lagerwall JPF, Giesselmann F. Current topics in smectic liquid crystal research. Chem Phys Chem. 2006;7:20-45.

[2] Clark NA, Lagerwall ST. Submicrosecond bistable electrooptic switching in liquid crystals. Appl Phys Lett. 1980;36:899-901.

[3] Takanishi Y, Ouchi Y, Takezoe H, et al. Chevron layer structure in the smectic-A phase of 8CB. Jpn J Appl Phys Lett. 1989;28:L487-L489.

[4] Rieker TP, Clark NA, Smith GS, et al. "Chevron” local layer structure in surface-stabilized ferroelectric smectic-C cells. Phys Rev Lett. 1987;59:2658-2661.

[5] de Vries A. Experimental-evidence concerning two different kinds of smectic-C to smectic-A transitions. Mol. Cryst Liq Cryst. 1977;41:27-31.

[6] de Vries A. Implications of the diffuse-cone model for smectic A-phase and C -phase and AC phase-transitions. Mol Cryst Liq Cryst. 1979;49:179-185.

[7] de Vries A. The description of the smectic A and C phases and the smectic A-C phase transition of TCOOB with a diffuse-cone model. J Chem Phys. 1979;71:25-31.

[8] de Vries A, Ekachai A, Spielberg N. Why the molecules are tilted in all smectic A phases, and how the layer thickness can be used to measure orientational disorder. Mol Cryst Liq Cryst. 1979;49:143-152.

[9] Diele S, Brand P, Sackmann H. X-ray diffraction and polymorphism of smectic liquid crystals 1 . A-, B- and C-modifications. Mol Cryst Liq Cryst. 1972;16:105-116.

[10] Clark NA, Bellini T, Shao RF, et al. Electro-optic characteristics of de Vries tilted smectic liquid crystals: analog behavior in the smectic $A^{\star}$ and smectic $C^{\star}$ phases. Appl Phys Lett. 2002;80:4097-4099.

[11] Shen Y, Wang L, Shao RF, et al. Generalized LangevinDebye model of the field dependence of tilt, birefringence, and polarization current near the de Vries smectic- $\mathrm{A}^{\star}$ to smectic- $\mathrm{C}^{\star}$ liquid crystal phase transition. Phys Rev E. 2013;88:062504.

[12] Selinger JV, Collings PJ, Shashidhar R. Field-dependent tilt and birefringence of electroclinic liquid crystals: theory and experiment. Phy Rev E. 2001;64:061705.

[13] Sreenilayam SP, Agra-Kooijman DM, Panov VP, et al. Phase behavior and characterization of heptamethyltrisiloxane-based de Vries smectic liquid crystal by electrooptics, $\mathrm{x}$ rays, and dielectric spectroscopy. Phys Rev E. 2017;95:032701.

[14] Sreenilayam SP, Rodriguez-Lojo D, Panov VP, et al. Design and investigation of de Vries liquid crystals based on 5-phenyl-pyrimidine and (R,R)-2,3-epoxyhexoxy backbone. Phys Rev E. 2017;96:042701.

[15] Yadav N, Panov VP, Swaminathan V, et al. Chiral smectic-A and smectic-C phases with de Vries characteristics. Phys Rev E. 2017;95:062704.

[16] Panarina OE, Panarin Yu P, Antonelli F et al. Investigation of de Vries $\mathrm{SmA}^{*}$ mesophases in low molecular weight organosiloxane compounds. J Mater. Chem. 2006;16:842-849.

[17] Kocot A, Vij JK, Perova TS, et al. Observation of the de Vries behavior in $\mathrm{SmA}^{\star}$ phase of a liquid crystal using polarised Raman scattering and infrared spectroscopy. J Chem Phys. 2017;147:094903.

[18] Hayashi N, Kato T, Fukuda A, et al. Evidence for de Vries structure in a smectic-A liquid crystal observed by polarized Raman scattering. Phys Rev E. 2005;71: 041705; Naciri J, Ruth J, Crawford G et al. Novel ferroelectric and electroclinic organosiloxane liquid crystals, Chem. Mater. 1995, 7: 1397-1402.

[19] Sreenilayam SP, Rodriguez-Lojo D, Agra-Kooijman DM, et al. de Vries liquid crystals based on a chiral 5-phenylpyrimidine benzoate core with a tri- and tetra-carbosilane backbone. Phys Rev Mater. 2018;2:025603.

[20] Park B, Seomun SS, Nakata M, et al. Collective molecular motion during $\mathrm{V}$-shaped switching in a smectic liquid crystal. Jpn J Appl Phys. 1999;38:1474-1481.

[21] Manna U, Song JK, Panarin YP, et al. Electro-optic and dielectric study of the de Vries-type smectic- $\mathrm{A}^{\star}$ phase exhibiting transitions to smectic- $\mathrm{C}_{\mathrm{A}}{ }^{*}$ and smectic- $\mathrm{C}^{\star}$ phases. Phys Rev E. 2008;77:041707.

[22] Swaminathan V, Panov VP, Panarin YP, et al. The effect of chiral doping in achiral smectic liquid crystals on the de Vries characteristics: smectic layer thickness, electro-optics and birefringence. Liq Cryst. 2018;45(4):513-521.

[23] Agra-Kooijman DM, Yoon HG, Dey S, and Kumar S. Origin of weak layer contraction in de Vries smectic liquid crystals, Phys. Rev. E. 2014;89:032506.

[24] P. Rudquist, Osipov MA and Geisselmann F. On the orientation distribution functions in de Vries type smectic liquid crystals. Liq. Cryst. 2018. doi.org/ $10.1080 / 02678292.2018 .1489985$ 